\title{
WELL-POSEDNESS AND REGULARITY OF THE CAUCHY PROBLEM FOR NONLINEAR FRACTIONAL IN TIME AND SPACE EQUATIONS
}

\author{
V. N. Kolokoltsov And M. A. Veretennikova
}

Abstract. The purpose is to study the Cauchy problem for non-linear in time and space pseudodifferential equations. These include the fractional in time versions of Hamilton-Jacobi-Bellman (HJB) equations governing the limits of controlled scaled Continuous Time Random Walks (CTRWs). As a preliminary step which is of independent interest we analyse the corresponding linear equation proving its well-posedness and smoothing properties.

Mathematics subject classification (2010): 35R11.

Keywords and phrases: fractional calculus, Caputo derivative, Mittag-Leffler functions, fractional Hamilton-Jacobi-Bellman type equations.

\section{REFERENCES}

[1] K. DieTheLm, The analysis of fractional differential equations, Springer, (2004).

[2] V. Kolokoltsov, Markov processes, semigroups and generators, De Gruyter, (2011).

[3] I. Podlubny, Fractional differential equations, An introduction to fractional derivatives, fractional differential equations, to methods of their solution and some of their applications, Mathematics in Science and engineering, Science and Engineering series 198, (1999).

[4] V. Kolokoltsov, Generalized continuous time random walks, subordination by hitting times and fractional dynamics, Probability Theory and Applications 53 (4), (2009).

[5] M. Veretennikova AND V. Kolokoltsov, A fractional Hamilton Jacobi Bellman equation for scaled limits of controlled Continuous Time Random Walks, Obtained in SIMAI CAIM Special Issue (2014), (2014).

[6] M. Veretennikova And V. Kolokolts ov, Scaling limits of controlled Continuous Time Random Walks and their position-dependent extensions, Preprint in preparation, 2014.

[7] V. Yu. Korolev and N. N. Skvortsova, Stochastic Models of Structural Plasma turbulence, VSP, (2006).

[8] V. M. Zolotarev, One-dimensional stable distributions, v. Translations of Mathematical Monographs, vol. 65, American Mathematical Society, Providence, (1986).

[9] M. Meerschaert And E. Nane And P. Vellais amy, Fractional Cauchy problems on bounded domains, The Annals of Probability 37 (3), 979-1007, (2009).

[10] E. Bajlekova, Fractional Evolution Equations in Banach Spaces, PhD thesis, (2001).

[11] L. KeXUE AND P. Jigen, Laplace transform and fractional differential equations, Appl. Math. Letters 24 (2011), 2019-2023.

[12] M. MATAR, On existence and uniqueness of the mild solution for fractional semilinear integrodifferential equations, Journal of integral equations and applications 23 (3), (2011).

[13] C. LizAmA AND G. M. N'GUEREKATA, Mild solutions for abstract fractional differential equations, Applicable Analysis 92 (8), 1731-1754, (2013).

[14] K. Diethelm, The analysis of fractional differential equations, An application-oriented exposition using differential operators of Caputo type, Springer, Lecture notes in Mathematics, (2004).

[15] Esra Alparslan, Classical and generalised Mittag-Leffler functions and applications, Graduate Project Presentation, Cancaya University, (2009).

[16] S. E. Eidelman AND A. N. KochuBEI, Cauchy problem for fractional differential equations, Journal of differential equations 199 (2004), 211-255. 
[17] Y. ZHOU AND X. N. SHEN AND L. ZHANG, Cauchy problem for fractional evolution equations with Caputo derivative, The European Physical Journal Special topics, EDP Sciences, Springer-Verlag 222 (2013), 1749-1765.

[18] Y. MA AND F. Zhang AND C. LI, The asymptotics of the solutions to the anomalous diffusion equations, Computers and Mathematics with Applications 66 (2013), 682-692.

[19] M. M. KoKURIn, The Uniqueness of a Solution to the Inverse Cauchy Problem for a Fractional Differential Equation in a Banach Space, Izvestiya Vysshikh Uchebnykh Zavedenii, Matematika 12 (2013), 19-35.

[20] A. V. PSKHU, Multi-time fractional diffusion equation, The European Physical Journal Special topics, EDP Sciences, Springer-Verlag 222 (2013), 1939-1950.

[21] A. A. Kilbas and M. Rivero and L. RodrigueZ-Germa And J. J. Trujillo, Caputo linear fractional differential equations, Proceedings of the 2nd FAC Workshop on Fractional Differentiation and Applications, Porto, Portugal, (2006).

[22] R. Gorenflo AND YU. F. LuChKo AND P. P. ZABRejKo, On solvability of linear fractional differential equations in Banach spaces, Fract. Calc. Appl. Anal. 2 (1999), 163-176.

[23] H. TAO AND M. FU AND R. QIAN, Positive solutions for fractional differential equations from real estate asset securisation via new fixed point theorem, Abstract Appl. Anal., (2012).

[24] G. SAmorodnits Ky And M. S. TAQQU, Stable non-Gaussian random processes: Stochastic models with infinite variance, Chapman and Hall CRC, (1994).

[25] Z. Q. Chen and M. M. Meerschaert And E. Nane, Space time fractional diffusion on bounded domains, Journal of Mathematical Analysis and Applications 393, (2012), 479-488.

[26] L. C. EVAnS AND R. F. GARIEPY, Measure theory and fine properties of functions, CRC Press, Inc., (1992).

[27] V. E. TARAsov, Fractional Dynamics, Applications of Fractional Calculus to Dynamics of Particles, Fields and Media, Springer, Higher Education Press, XV, (2011).

[28] V. V. UChaikin, Fractional Derivatives for Physicists and Engineers, Springer, (2012).

[29] E. Scalas, R. Gorenflo, F. Mainardi And M. M. Meerschaert, Speculative option valuation and the fractional diffusion equation, In: A. Le Méhauté, J. A. Tenreiro Machado, J. C. Trigeassou and J. Sabatier (Editors). Fractional Differentiation and its Applications. U-Books (2005), pp. 265-274. [Selected papers of the First IFAC Workshop on Fractional Differentiation and Applications (FDA'04), Bordeaux (France) 19-21 July 2004], (2004).

[30] J. A. Tenreiro Machado and M. S. F. Alexandra Galhano, Fractional Order Inductive Phenomena Based on the Skin Effect, Nonlinear Dynamics 68 (1-2), 107-115, (2012).

[31] V. V. Anh And C. C. Heyde AND N. N. LeOnenko, Dynamic models of long-memory processes driven by Lévy noise, J. Appl. Probab. 39 (4), 671-913, (2002). 